\title{
Saving emergency medicine: is less more?
}

\author{
Paul Atkinson $^{1}$ [D $\cdot$ Ken McGeorge ${ }^{2} \cdot$ Grant Innes $^{3}$
}

Received: 26 October 2021 / Accepted: 16 November 2021

(C) The Author(s), under exclusive licence to Canadian Association of Emergency Physicians (CAEP)/ Association Canadienne de Médecine d'Urgence (ACMU) 2021

Keywords Emergency medicine $\cdot$ Primary care $\cdot$ Crowding

\section{The problem}

Emergency departments are failing. Triage lineups, packed waiting rooms, ambulance offload delays, unacceptable treatment delays, waiting room disasters and frustrated patients leaving before they are seen. Add COVID to the mix: long hot days in masks and PPE. Aerosol-generating procedures on critical patients. Ventilated patients and "screened positive" patients blocked in the ED waiting for non-existent ICU or isolation beds. Dangerous daily exposures for staff, susceptible patients and their families. Nurses hitting the wall, leaving emergency departments for more sustainable lives, with growing staff shortages further depleting ED capacity. A vicious cycle of demand, dysfunction and distress that aggravates crowding, wait times and left without being seen rates $[1-3]$.

Emergency departments need repair. Or do they? A report to the Canadian Foundation for Healthcare Innovation shows that ED visits are increasing much faster than population growth and that, without fundamental system change, they will grow an additional $40 \%$ by 2043 [4]. Canadians depend more and more on emergency departments for care they cannot get elsewhere, and Canadians have the highest rate of ED utilization in the first world [5]. Canada performs worst among OECD countries in providing access to primary care, specialists, surgical procedures and imaging [5, 6]. Many Canadians cannot get a family physician. Those who have

Paul Atkinson

Paul.atkinson@dal.ca

1 Department of Emergency Medicine, Dalhousie University, Horizon Health Network, Saint John Regional Hospital,

University Ave, Saint John, NB E2L 4L2, Canada

2 Health Administration Consultant, Fredericton, NB, Canada

3 Department of Emergency Medicine, University of Calgary, Calgary, AB, Canada one can rarely get same-day, next-day or after-hours appointments, so emergency departments have, by default, become major providers of community and primary care $[5,6]$.

And it is not just primary care. When long-term care facilities cannot manage elderly residents, they are sent to EDs, not because we have geriatric expertise but because we will see the patient today. Community physicians who need an urgent surgical or specialist consult send their patients instead to an ED because there are no urgent specialty referral pathways. Surgical patients are told (you guessed it) to go to the emergency department if they develop a post-op problem [6]. Poor and marginalized patients are disproportionately treated in EDs, and half of these visits are for non-urgent concerns [6]. Patients facing prolonged delays for specialist appointments or imaging studies frequently head for emergency departments when they deteriorate or become frustrated. When inpatient programs reach capacity and cannot manage their patients, EDs are left holding the bag for large numbers of admitted medical, surgical and mental health patients who should be in hospital beds. These patients often occupy the bulk of ED stretcher spaces, decimating the ability to provide actual emergency care.

Accessibility is one of the five core principles of the Canada Health Act, but there is only one open door for Canadians who fail to plan their illness or injury. The ED is the default destination for almost all unscheduled care, and $58-80 \%$ of ED patients went to an ED because it was the only place they could get care when they needed it [7]. Research confirms that the unbridled demand facing emergency departments arises from poor primary care accessibility, increasing patient complexity, a rising burden of unmanaged chronic disease, physician and nurse staffing shortages, and a lack of hospital beds for admitted patients $[6,8]$. None of these factors fall within an emergency medicine sphere of influence. So, is it really emergency departments that need fixing? 
The emergency medicine credo is that every patient's concern is important and that patients cannot be turned away, regardless of their condition. Our attempts to provide unconditional service have paved the way for other providers to eschew unplanned illness and injury, optimize their schedules, avoid inconvenient disruptions in alwaysbusy days, and address countless inconvenient patient needs with a simple almost magical directive: "Go to the emergency department." But, can EDs fill the care gaps left by other programs and simultaneously provide rapid, high-quality emergency care? The answer is no. The concept of the ED as healthcare's universal contingency plan is flawed and dangerous. We do our best, but rising volumes, complexity and stress levels, the toll of emergency providers leaving the field, and ever-increasing demands to deliver inpatient care, primary care and non-emergent care that should be provided elsewhere have become an unmanageable load. Our efforts to carry this load have left us failing, frustrated and wasting by attrition.

The ED is the wrong place for most patients. Excessive and inappropriate use of emergency departments increases system cost, decreases care quality and creates chaotic unpleasant work environments that burn out staff [6]. EDs are designed for 1-6-h encounters. Emergency teams are trained and equipped for acute problems and life-limb threats. We are not psychiatrists, surgeons, geriatricians or GPs. We do not provide high quality inpatient care, mental health intervention, chronic disease management, rehabilitation services, or primary and preventive health care. Leaving frail or acutely ill patients on hard narrow stretchers in noisy crowded rooms where the lights never go out, without privacy, sleep, or bathroom access while they wait hours or days for a hospital bed is not acceptable anywhere else in the healthcare system. Why is it acceptable here?

With admitted patients filling many or most ED stretchers, truly emergent patients are often blocked outside. To prevent waiting room disasters, ED physicians now assess patients in hallways and waiting rooms, but with a quagmire of undifferentiated, unmanaged, chronically unwell and frustrated patients at the front door, our attention is increasingly diverted from the diminishing proportion of high-risk patients hidden in the crowd. It is a veritable "Where's Waldo" exercise every day in ED waiting rooms. Concealed in the queue are unrecognized time bombs: abdominal pains with ruptured ectopics, leg pains with necrotizing fasciitis and headaches with subarachnoid hemorrhage. These are seldom identifiable during a triage encounter and their care delays too frequently cause disastrous outcomes, media headlines and government commissions that repeatedly fail to determine the cause of emergency department dysfunction.

\section{The solution?}

Perhaps it is time to rethink "emergency"? With rising ED demand, access failures throughout the system, and mounting staff shortages, emergency departments can no longer cope. In this broken system, we have to clarify our primary mission, redefine what an emergency patient is, and reimagine our processes. Perhaps we should limit our focus to acute injuries, emergencies and lifesaving care in keeping with the specialty's original intent. But, this would require that many current ED patients have appropriate care alternatives. It would mean asking family physicians and specialists to provide better access to unscheduled and off-hours care [6, 9].

Patients deserve the right care from the right providers. Urgent care centres could manage many non-severe injuries and illnesses, and manage unattached patients. Family physicians could assure same or next day care options. Surgeons could accept urgent community referrals and expedite unscheduled clinic checks for post-op problems. Long-term care facilities could improve after-hours and weekend coverage to reduce ED transports, and community physicians should have better access to imaging and specialty referral to avoid unnecessary low-value ED visits. Most important, hospitals could develop contingencies to move inpatients to inpatient care units in a timely fashion.

It seems presumptuous for emergency leaders to propose change in other programs. Unfortunately, other programs have developed convenient solutions that profoundly impact emergency departments. The concepts above reflect an understanding that the best outcomes occur when patients receive the right care from the right provider, and that all programs are accountable to assure access for their populations [10].

Crises are also opportunities and emergency medicine should seize this moment. Because most root causes and solutions lie outside our departments [6, 11], emergency leaders must drive change within and beyond the ED. The first step is cleaning up our own back yard. Asking others to implement difficult change only works if we are willing to do the same. ED improvements are necessary but not sufficient, and access initiatives limited to the ED will likely have adverse consequences. EDs that have improved efficiency and freed up stretcher capacity have often seen inpatient services rapidly consume that capacity by boarding even more inpatients for longer times in ED stretchers. Bailing out only one end of the boat is not a recipe for success, and ED improvement without system change may have negative consequences for our patients, our staff and ourselves [12].

In addition to operational and system change, it may be time to acknowledge and promote the distinction between 
emergency medicine, urgent care, primary care, and family medicine. We might consider limiting our care to "appropriate" patients, as other programs have done. This could dramatically simplify our lives and make our primary mission feasible, but unless other programs pick up the slack, this action will have detrimental effect on patients. The key solution is to establish accountability frameworks that specify accountability zones, access targets, surge contingency plans, and queue management expectations for all programs. A system without accountability will not succeed $[9,10]$.

There are other approaches to unscheduled care. Perhaps home visits, the medical equivalent of Skip the dishes, will re-emerge. Perhaps virtual health visits and telemedicine will save the day. However, while these are great for prescription refills and stable patients, a shift away from faceto-face care could mean even more ED visits for patients who actually have something wrong with them that cannot be diagnosed by phone.

The system will not fix itself, and our future is what we make it. Emergency physicians who want a better future for their departments, their nursing colleagues and their patients, must lead system change. Others (who depend on emergency departments to respond to all forms of patient need) are unlikely to envision solutions that make their own lives more difficult. But, as long as we are doing our best, we must practice self-compassion when we feel pangs of guilt that we cannot meet every patient's needs, every day.

\section{Declarations}

Conflict of interest All authors confirm that they have no conflicts of interest to declare.

\section{References}

1. Grant K. Canadian nurses are leaving in Droves, worn down by 16 merciless months on the front lines of COVID-19. In: The Globe and Mail. 2021. https://www.theglobeandmail.com/canada/articlecanadian-nurses-are-leaving-in-droves-worn-down-by-16-merci less-months/. Accessed 10 Sep 2021
2. Sutherland M, Ibrahim H. Oromocto hospital ER to CLOSE 6 hours earlier each day due to staff SHORTAGE I CBC News. In: CBCnews. 2021; https://www.cbc.ca/news/canada/new-bruns wick/overnight-er-closure-doctor-shortage-1.6073531. Accessed 10 Sep 2021

3. Lam P. Staff raise alarm Over 'exceptionally dangerous' wait times at St. Boniface Hospital I CBC News. In: CBCnews. 2021; https:// www.cbc.ca/news/canada/manitoba/16-hour-wait-time-st-bonif ace-hospital-staff-shortage-1.6135667. Accessed 10 Sep 2021

4. Barr S, Campbell S, Flemons W et al. The Impact on Emergency Department Utilization of the CFHI Healthcare Collaborations and Initiatives: Report to the Canadian Foundation for Healthcare Improvement. 2013; pp. 2-44. https://www.cfhi-fcass.ca/ docs/default-source/about-us/corporate-reports/risk-analytica. pdf?sfvrsn=f2ef76ab 2. Accessed Oct. 15, 2021

5. Canadian Institute for Health Information (CIHI). How Canada Compares: Results from the Commonwealth Fund 2016 International Health Policy Survey. Ottawa, ON; 2016. https://www. cihi.ca/sites/default/files/document/text-alternative-version-2016cmwf-en-web.pdf. Accessed October 14, 2021

6. Berchet C. The Directorate for Employment, Labour and Social Affairs Health Committee of the Organisation for Economic Cooperation and Development (OECD). Emergency care services: Trends, drivers and interventions to manage the demand. 2015. https://www.oecd-ilibrary.org/docserver/5jrts344crns-en.pdf? expires $=1634347017 \& \mathrm{id}=\mathrm{id} \&$ ac name $=$ guest $\&$ checksum $=$ 006E58338F7FD4DBEA29CC332496DF2F. Accessed October 6,2021

7. Health Quality Council of Alberta. Review of the quality of care and safety of patients requiring access to emergency department care. 2012. https://hqca.ca/wp-content/uploads/2018/05/EDCAP_ FINAL_REPORT.pdf . Accessed October 7, 2021

8. Rowe $\mathrm{BH}$, Bond $\mathrm{K}$, Ospina MB, et al. Emergency department overcrowding in Canada: what are the issues and what can be done. Canadian Agency for Drugs and Technologies in Health. Technology overview. 2006.

9. Atkinson P, Innes GD. Patient care accountability frameworks: the key to success for our healthcare system. Can J Emerg Med. 2021;23:274-6.

10. Innes GD. Sorry—we're full! Access block and accountability failure in the health care system. Can J Emerg Med. 2015;17:171-9.

11. Morley C, Unwin M, Peterson GM, et al. Emergency department crowding: a systematic review of causes, consequences and solutions. PLoS ONE. 2018;13(8):e0203316.

12. Lim R, Van Aarsen K, Gray S, Rang L, Fitzpatrick J, Fischer L. Emergency medicine physician burnout and wellness in Canada before COVID 19: a national survey. Can J Emerg Med. 2020;22(5):603-7. 